\title{
Classification des cirrhoses
}

Le $5^{\circ}$ Congrès pan-amêrícaín de gastro-entêrologie, réuni à Cuba du 20 au 27 juin 1956, avait désigné une commission chargée d'établir une classification des cirrhoses hépatiques. L'Europe était représentee dans cette commission de 6 membres par la présidente, le Dr Sheila

\section{Editorial}

801

Sherlock, de Londres, et par le Dr H. Popper, viennois d'origine, élève å”Eppinger. II vaut la peine de résumer ici les conclusions de ces travaux, rapportés dans Gastroenterology d'août 1956 (vol. 31, p. 213).

$1^{\circ}$ La definition de la cirrhose hépatique est essentíellement ana-tomíque; elle comporte une atteinte diffuse de la glande, avec necrose cellulaire, regeneration parenchymateuse et fibrose bouleversant $\Gamma$ architecture lobulaire. A cette definition histologique s'ajoute une note clinique, distinguant la maladie chronique de la decompensation («liver cell failure»), avec ou sans hypertension portale.

$2^{\circ}$ La classification sera établie sur la base des facteurs suivants:

morphologíques: cirrhoses portale, post-nécrotique ou biliaire;

êtíologíques, parnai lesquels le role de la malnutrition, de Гalcool, d'un virus, de la cholostase, de $\Gamma$ insuffisance cardique, de $\Gamma$ hémo-chromatose est admis; tandis que $\Gamma$ influence d'agents toxiques, d'in-fections telles que la tuberculose et la brucellose, d'infestations para-sitaires, est contestée;

fonctíonnels, soit: insuffisance parenchymateuse (ictère, ascite, hypoprotidémie, etc.); hypertension portale (splénomégalie, varices cesophagiennes, etc.); tendance evolutive (progressive, stationnaire, regressive) de la maladie.

La combínaíson de ces trois facteurs permet une nomenclature nuancée tenant compte des différentes formes observées en clinique, comme par exemple:

Cirrhose portale progressive, d'origine alcoolique, avec insuffisance hépatique, mais sans hypertension portale.

Cirrhose post-nécrotique, faisant suite à une hepatite à virus, sans insuffisance hépatique mais avec hypertension portale, à evolution favorable (regressive).

Cirrhose biliaire progressive, consecutive à un rétrécissement cholé-docien, sans insuffisance hépatique ni hypertension portale.

La Commission estime done que le diagnostic complet d'une cirrhose doit mentionner successivement: a) la morphologie (éven-tuellement d'après la ponction-biopsie); b) l'origine de la maladie; c) son aspect fonctionnel et d) sa tendance evolutive. Si nos spécialistes européens peuvent se rallier à cette conception, et que la terminologie des publications mondiales voulait bien se conformer à ces directives, il en résulterait sans doute une meilleure comprehension, dans un domaine touffu où les mots comportent des valeurs différentes selon les auteurs. Les definitions de la conference de Cuba ne sont d'ailleurs

802

Editorial 
pas definitives, et une premiere revision est prévue lors du Congrès international de Washington en 1958. Car les membres de la commis sion ont prononcé $\Gamma$ exclusion d'une série d'expressions courantes dans le langage medical français, telles notamment: cirrhoses atrophique ou hypertrophique; cirrhose pigmentaire; cirrhose de Hanot; cirrhose alcoolique, et cirrhose post-hépatitique - qui ne devraient plus être utilisées, d'après les conclusions des experts de Cuba. M. D. Seite der Therapie - Page de thérapeutique Richman, A., I. Sternlíeb and A. Wirikelstein: ACTH and Cortisone Therapy in Ulcerative Colitis. Amer. J. Dig. Dis. (New Series) J, 206-214 (May 1956).

27 malades atteints de côlite ulcéreuse qui n'avaient pas été améliorés par un traitement medical classique ont été traités à $\Gamma \mathrm{ACTH}$, à la Cortisone ou a $\Gamma$ Hydro-cortisone.

Ces stéroïdes ont permis de hater ou de provoquer la remission dans 21 cas sur 34, mais on n'a pas observe d'effet curatif. Les manifestations extra-intestinales de la côlite ulcéreuse (érythème noueux, arthrite, conjonctivite) ont réagi plus rapide-ment à cette thérapeutique que la maladie intestinale elle-même. Aucun cas de mort ne survint en cours de traitement; par centre il faut signaler deux perforations et une hémorragie severe.

9 malades ont eu recours finalement à la chirurgie, en raison de la resistance de leurs symptômes et du manque de réponse à la thérapeutique rnédicale y compris les stéroïdes. B. Wíssmer Solem, J. H., 0. Knutrud et Kr. Andresen: Traitement de la pancréatite aiguë par l'ACTH. Acta Chirurgica Scandinavica 109, 415-420 (oct. 1955).

Observation d'une femme de 83 ans chez laquelle le diagnostic de pancréatite aiguë a été porté par des signes cliniques, radiologiques et biologiques.

100 U.I. d'ACTH eurent un effet immédiat, et associée aux antibiotiques, fut poursuivie à cette dose pendant 3 jours et dut être reprise, au bout de 3 jours égale-ment, en raison d'une nouvelle poussée evolutive.

La courbe de $\Gamma$ amylasurie, très élevée au debut, tomba sous $\Gamma$ effet de $\Gamma A C T H$, mais se relèva avec la rechute pour redescendre à nouveau avec la reprise du traitement.

Mallet-Guy, P., J. Féroldi et Eicholz: L'évolution des lesions d'hépatite après neurectomie périartère hépatique. Lyon Chir. 49, 297-312 (1954).

L'intervention a été réalisée chez 40 malades; sa bénignité paraît certaine.

Dans 18 cas d'íctère chronique, le prurit disparaît dès le premier jour. Les selles se recolorent, Tictère rétrocède progressivement. Les résultats fonctionnels sont très favorables et se maintiennent, dans certains cas, depuis 4 ans. La condition 